\title{
Addendum: Anomalous mechanical behavior of the deltic, squaric and croconic cyclic oxocarbon acids
}

\author{
Francisco Colmenero ${ }^{1}$ \\ ${ }^{1}$ Molecular Physics Department, Instituto de Estructura de la Materia (CSIC), Madrid, Spain \\ E-mail: francisco.colmenero@iem.cfmac.csic.es
}

Received xxxxxx

Accepted for publication $\mathrm{xxxxxx}$

Published xxxxxx

\begin{abstract}
The mechanical properties of the deltic, squaric, and croconic acids were determined in a previous work by using rigorous theoretical solid-state methods based in Density Functional Theory using plane waves and pseudopotentials using a high-quality setup. These three materials were shown to display small negative Poisson ratios (NPR). Croconic acid was also shown to exhibit the phenomenon of negative linear compressibility (NLC) for applied pressures larger than $\sim 0.4 \mathrm{GPa}$ directed along the direction of minimum Poisson ratio. The main purpose of this communication is to show that the croconic acid also exhibits the NLC phenomenon when submitted to isotropic pressures. The $a$ lattice parameter of croconic acid increases for applied isotropic pressures in the range from $0.41 \mathrm{GPa}$ to $0.46 \mathrm{GPa}$. The computed compressibility along $a$ direction at the pressure of $\mathrm{P}=0.44 \mathrm{GPa}$ is $k_{a}=-79.7 \mathrm{TPa}^{-1}$.
\end{abstract}

Keywords: croconic acid, negative linear compressibility, DFT

\section{Introduction}

The compressibility is a mechanical property which measures the relative volume change of a material as a response to a pressure change and must be strictly positive for solid materials which are thermodynamically and mechanically stable [1-4]. A material submitted to a hydrostatic (isotropic) compressive pressure cannot increase its volume, unless it is unstable. Negative linear compressibility (NLC) is not related to unstable materials and may refer to $(a)$ the positive variation of the volume when the material is submitted to a uniform compression directed along a given direction and $(b)$ the increase of one or two single directions of the unit cell of a material under the application of a hydrostatic pressure [1-3]. In (a) the directional derivative of the volume with respect to pressure is negative. In $(b)$ the volume does not increase and the positive variation of the parameters in some directions is compensated by a larger negative variation of the parameters in the remaining directions.
In a previous paper [5], the mechanical properties of the deltic, squaric, and croconic acids were determined by using theoretical solid-state methods based in Density Functional Theory using plane waves and pseudopotentials. The energy-density functional employed was the PBE [6] one supplemented with Grimme [7] empirical dispersion correction. This functional is implemented in CASTEP [8] code, a module of the Materials Studio [9] software, which was used in all the computations. These three materials were shown to display small negative Poisson ratios (NPR) [4]. The croconic acid also exhibits the phenomenon of negative linear compressibility (NLC) [1-3] for applied pressures larger than about $0.4 \mathrm{GPa}$ directed along the direction of minimum Poisson ratio. The same methods were employed in this communication with the purpose of showing that the croconic acid also exhibits NLC when submited to isotropic pressures. The details of the computational solid-state treatment of the oxocarbon acids used in this work may be found in the previous paper [5]. 
Table 1. Unit cell volume and lattice parameters of the croconic acid under different isotropic pressures.

\begin{tabular}{ccccc}
\hline $\mathbf{P}(\mathbf{G P a})$ & $\boldsymbol{a}(\mathbf{\AA})$ & $\boldsymbol{b}(\mathbf{\AA})$ & $\boldsymbol{c}\left(\AA^{\mathbf{A}}\right)$ & Vol. $_{\left(\AA^{\mathbf{3}}\right)}$ \\
\hline 0.261 & 8.6824 & 5.0486 & 10.7856 & 472.7784 \\
0.371 & 8.6679 & 5.0362 & 10.7760 & 470.4077 \\
0.422 & 8.6561 & 5.0281 & 10.7747 & 468.9529 \\
0.453 & 8.6754 & 4.9986 & 10.7687 & 466.9833 \\
0.477 & 8.6749 & 4.9983 & 10.7682 & 466.9025 \\
0.526 & 8.6604 & 4.9973 & 10.7660 & 465.9413 \\
0.567 & 8.6489 & 4.9953 & 10.7625 & 464.9864 \\
0.595 & 8.6434 & 4.9943 & 10.7605 & 464.5067 \\
0.622 & 8.6405 & 4.9944 & 10.7588 & 464.2844 \\
0.683 & 8.6364 & 4.9863 & 10.7530 & 463.0603 \\
\hline
\end{tabular}
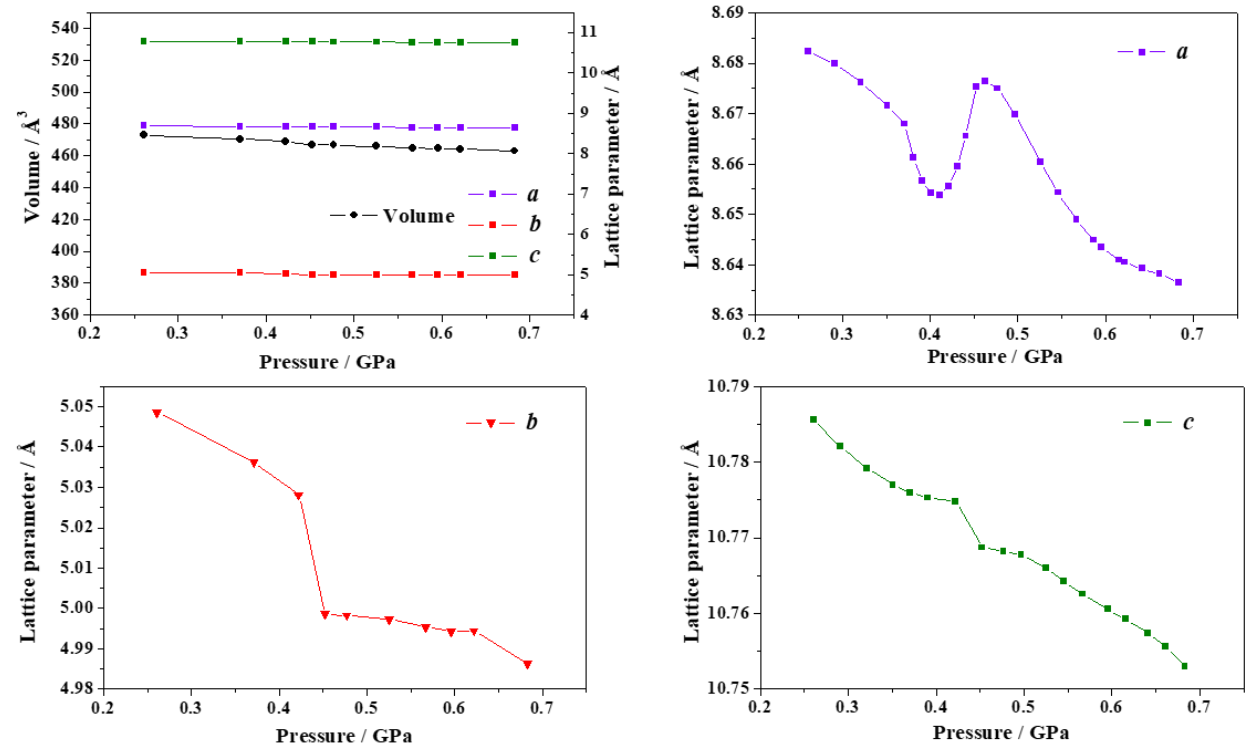

Figure 1. Unit cell volume and lattice parameters of the croconic acid under the effect of different isotropic pressures.

\section{Results}

Table 1 reports the computed unit cell volumes and lattice parameters of the solid croconic acid submitted to different isotropic pressures. The results are also displayed in Figure 1. As can be observed in Figure 1, the $a$ lattice parameter of this material increases under the application of hydrostatic pressures in the range from $0.41 \mathrm{GPa}$ to $0.46 \mathrm{GPa}$. The large increase of the $a$ lattice parameter is accompanied by a large decrease of $b$ lattice parameter. The computed linear compressibility at 0.44 $\mathrm{GPa}$ is $k_{a}=-1 / a \cdot(\partial a / \partial P)_{P}=-79.7 \mathrm{TPa}^{-1}$. From the inspection of deltic and squaric acid data, these materials appear not to show any sign of isotropic NLC.

\section{Conclusions}

While the range of pressure for which the solid croconic acid shows isotropic NLC is quite narrow (from $0.41 \mathrm{GPa}$ to $0.46 \mathrm{GPa})$, the magnitude of the negative compressibility is quite large. The value, $79.7 \mathrm{TPa}^{-1}$, is one of the largest found so far. The largest value of the
NLC, about -260 $\mathrm{TPa}^{-1}$, was found for $\mathrm{CsH}_{2} \mathrm{PO}_{4}$ [10-11] and is much larger than the present value. However, the compressibilities found for materials as $\mathrm{Zn}\left[\mathrm{Au}(\mathrm{CN})_{2}\right]_{2}$ [12] and $\mathrm{Ag}_{3}\left[\mathrm{Co}(\mathrm{CN})_{6}\right]$ [13-14], -42 and $-76 \mathrm{TPa}^{-1}$, considered to be very large, are of the same magnitude as that found in this work for croconic acid. While the range of external pressures in which these materials show NLC is much larger than in croconic acid, the range is extended if anisotropic pressures are applied to croconic acid along the direction of the minimum Poisson ratio [5]. As showed in the previous work, croconic acid displays anisotropic NLC for pressures larger than about $0.4 \mathrm{GPa}$ up to about $1.0 \mathrm{GPa}$, when this material undergoes a pressure induced phase transition.

\section{Acknowledgements}

Supercomputer time by the CTI-CSIC center is greatly acknowledged. I want to thank Dr. A. M. Fernández for reading the manuscript and many helpful comments. 


\section{References}

[1] Baughman R H, Stafström S, Cui C and Dantas S O 1998 Materials with Negative Compressibilities in One or More Dimensions Science 279, 1522-1524

[2] Kornblatt J A, Sirota E B, King H E, Baughman R H and Cui C 1988 Materials with Negative Compressibilities Science 281, 143-143

[3] Cairns A B and Goodwin A L 2015 Negative Linear Compressibility Phys. Chem. Chem. Phys. 17, 20449-20465

[4] Lakes R S 2017 Negative-Poisson's-Ratio Materials: Auxetic Solids Annu. Rev. Mater. Res. 47, 63-81

[5] Colmenero F 2018 Anomalous mechanical behavior of the deltic, squaric and croconic cyclic oxocarbon acids Mater. Res. Express Under Review

[6] Perdew J P, Burke K and Ernzerhof M 1996 Generalized Gradient Approximation Made Simple Phys. Rev. Lett. 77, 3865-3868

[7] Grimme S 2006 Semiempirical GGA-type Density Functional Constructed with a Long-Range Dispersion Correction $J$. Comput. Chem. 27, 1787-1799

[8] Clark S J, Segall M D, Pickard C J, Hasnip P J, Probert M I J, Refson K and Payne M C 2005 First Principles Methods Using CASTEP Z. Kristallogr. 220, 567-570

[9] MaterialsStudio, http://3dsbiovia.com/products/collaborativescience/biovia-materials-studio/ (accessed September 27, 2018)

[10] Prawer S, Smith T F and Finlayson T R 1985 The Room Temperature Elastic Behaviour of $\mathrm{CsH}_{2} \mathrm{PO}_{4}$ Aust. J. Phys. 38, 63-83.

[11] Kobayashi Y, Deguchi K, Azuma S, Suzuki E, Ming L C, Endo $\mathrm{S}$ and Kikegawa T 2003 Phase Transitions in $\mathrm{CsH}_{2} \mathrm{PO}_{4}$ Under High-Pressure Ferroelectrics 285, 83-89.

[12] Cairns A B, Catafesta J, Levelut C, Rouquette J, van der Lee A, Peters L, Thompson A L, Dmitriev V, Haines J and Goodwin A L 2013 Giant negative linear compressibility in zinc dicyanoaurate Nat. Mater. 12, 212-216.

[13] Goodwin A L, Calleja M, Conterio M J, Dove M T, Evans J S O, Keen D A, Peters L and Tucker M G 2008 Colossal Positive and Negative Thermal Expansion in the Framework Material $\mathrm{Ag}_{3}\left[\mathrm{Co}(\mathrm{CN})_{6}\right]$ Science 319, 794-797.

[14] Goodwin A L, Keen D A and Tucker M G 2008 Large negative linear compressibility of $\mathrm{Ag}_{3}\left[\mathrm{Co}(\mathrm{CN})_{6}\right]$ Proc. Natl. Acad. Sci. U. S. A. $105,18708-18713$. 